\title{
Rastreamento de risco de desfechos desfavoráveis em idosos: 0 instrumento Probabilidade de Internações Repetidas
}

\author{
Mariangela Perez ${ }^{1 *}$
}

\section{Resumo}

Introdução: Os sistemas de saúde sofrem uma forte pressão com o envelhecimento populacional e a consequente intensificação do uso dos serviços de saúde. A identificação dos idosos sob risco de fragilização é importante no manejo dessa população. Instrumentos breves de predição de risco para medir desfechos específicos são úteis para a identificação de indivíduos de alto risco. O instrumento Probabilidade de Internações Repetidas (Pir) foi proposto para o rastreamento de idosos com alto risco de internações hospitalares. Objetivos: Provocar uma reflexão sobre a utilidade do Pir a partir dos dados sobre a sua acurácia. Métodos: Revisão narrativa de estudos originais internacionais e brasileiros que utilizaram o Pir. Discussão: No estudo original, a acurácia do Pir foi de $61 \%$ para internações repetidas. A sensibilidade e especificidade foram de $12,7 \%$ e $94,6 \%$, respectivamente. Os valores preditivo positivo e negativo foram de $42 \%$ e $78 \%$, respectivamente. Em 2013, uma metanálise estimou a acurácia de 69,7\%, revelando uma modesta capacidade de discriminação do modelo, além de uma alta especificidade (95\%). Um estudo brasileiro longitudinal de validação preditiva do Pir apresentou a acurácia de 67\%. Conclusões: Constatou-se que, nos estudos que avaliaram as características psicométricas do Pir, a sensibilidade mostrou-se baixa, a despeito de alta especificidade e que, até o momento, não existem muitas evidências de seu desempenho na população brasileira. Embora utilizado há algumas décadas, a aplicação do Pir isoladamente para rastreio de idosos com risco de uso elevado dos serviços de saúde tem se mostrado inadequado, especialmente devido à baixa sensibilidade.

Descritores: Programas de rastreamento; Acurácia dos dados; Envelhecimento; Serviços de saúde.

\section{Abstract}

Risk screening for negative outcomes in old persons: The instrument Probability of repeated admission

Health systems are under severe pressure from population aging and the consequent increase in the use of health services. The identification of the old persons under risk of frailty is important in the management of this population. Brief risk prediction tools to measure specific outcomes are useful for identifying high-risk individuals. The instrument Probability of Repeated Admission (Pra) was proposed for the screening of older patients with high risk of hospital admissions. Objectives: to provoke a reflection on the utility of Pra from the data on its accuracy. Methods: Narrative review of original international and Brazilian studies using Pra. Results: In the
1. Laboratório de Envelhecimento Humano - GeronLab. Faculdade de Ciências Médicas. Universidade do Estado do Rio de Janeiro. Rio de Janeiro, RJ, Brasil.

*Endereço para correspondência:

Mariangela Perez

Rua Uruguai, 527/804

Rio de Janeiro, RJ. CEP: 20510-060.

E-mail: mariangela.perez@hotmail.com

Revista HUPE, Rio de Janeiro, 2017;16(2):110-116

doi: 10.12957/rhupe.2017.37655

Recebido em 25/07/2018. Aprovado em 01/08/2018.

original study, the accuracy of the Pra was $61 \%$ for repeated admission. The sensitivity and specificity were $12.7 \%$ and $94.6 \%$, respectively. The positive and negative predictive values were $42 \%$ and $78 \%$, respectively. In 2013, a meta-analysis estimated the accuracy of $69.7 \%$, revealing a modest capacity of discrimination of the model, in addition to a high specificity (95\%). A longitudinal Brazilian predictive validation study of Pra presented the accuracy of $67 \%$. Conclusion: It was observed that in the studies evaluating the psychometric characteristics of Pra, the sensitivity was low, despite the high specificity and that, for the moment, there is not much evidence of its performance in the Brazilian population. However used for a few decades, the application of Pra alone for screening old individuals at high risk of health care use has been inadequate, especially due to the low sensitivity..

Keywords: Mass screening; Data accuracy; Aging; Health services.

\section{Resumen}

Tamizaje de riesgo de resultados desfavorables en ancianos: El instrumento Probabilidad de internaciones repetidas

Los sistemas de salud sufren una fuerte presión con el envejecimiento de la población y la consiguiente intensificación del uso de los servicios de salud. La identificación de los ancianos bajo riesgo de fragilización es importante en el manejo de esa población. Los instrumentos breves de predicción de riesgo para medir resultados específicos son útiles para la identificación de individuos de alto riesgo. El instrumento Probabilidad de Internaciones Repetidas (Pir) fue propuesto para el rastreo de ancianos con alto riesgo de internaciones hospitalarias. Objetivos: Provocar una reflexión sobre la utilidad del Pir a partir 
de los datos sobre su exactitud. Métodos: Revisión narrativa de estudios originales internacionales y brasileños que utilizaron el Pir. Resultados: En el estudio original, la exactitud del Pir fue del $61 \%$ para internaciones repetidas. La sensibilidad y la especificidad fueron del $12,7 \%$ y del $94,6 \%$, respectivamente. El valor predictivo positivo y negativo fue del $42 \%$ y del $78 \%$, respectivamente. En 2013, un meta análisis estimó la exactitud del $69,7 \%$, revelando una modesta capacidad de discriminación del modelo, además de una alta especificidad (95\%). Un estudio brasileño longitudinal de validación predictiva del Pir presentó la exactitud del 67\%. Discusión: En los estudios

\section{Introdução}

A transição epidemiológica ocorre no Brasil como consequência da transição demográfica e resulta na mudança dos padrões de ocorrência de morbidade e de mortalidade. Há um aumento de doenças crônicas não transmissíveis (DCNT) somando-se às doenças infecciosas, potencialmente curáveis, ainda prevalentes em nosso meio. Essa mudança no perfil de morbidade da população, que surge como consequência da compressão da mortalidade, acarreta o aumento da proporção de indivíduos portadores de graus variados de incapacidade física ou mental. ${ }^{1}$ Diante desse panorama, a autonomia e a independência são aspectos centrais quando se planejam intervenções de saúde para indivíduos idosos. Portanto, diferentes níveis de ações tornam-se necessários para oferecer atenção integral e apropriada, de acordo com a trajetória funcional da pessoa, o que determina o aumento da complexidade das ações em saúde. Nesse novo cenário, o modelo atual, centrado no diagnóstico e cura de doenças, mostra-se anacrônico e insuficiente para as reais necessidades dessa parcela da população. ${ }^{2}$

Os sistemas de saúde sofrem uma forte pressão ocasionada pelo envelhecimento populacional e a consequente intensificação do uso dos serviços. No Brasil, o gasto com a saúde pública per capita em relação ao Produto Interno Bruto (PIB) dobrou na última década (2000-2010), e, em 2010, equivalia a 9,0\% do PIB. Os fatores determinantes para esse aumento de gastos são a transição epidemiológica, o uso de alta tecnologia e o cuidado concentrado no ambiente hospitalar. ${ }^{3}$ No Brasil, os hospitais estão no centro do cuidado e consomem em torno de 70\% dos recursos destinados ao Sistema de Saúde, a despeito da alta demanda por cuidados de longa duração para as doenças crônicas. Segundo o Banco Mundial, ${ }^{3}$ o tratamento das DCNT responde por cerca de metade dos gastos hospitalares. Em 2013, Silveira e colaboradores ${ }^{4}$ avaliaram as internações hospitalares que evaluaron las características psicométricas del Pir, la sensibilidad se mostró baja, a pesar de alta especificidad y que, hasta el momento, no existen muchas evidencias de su desempeño en la población brasileña. Aunque se utilizó hace algunas décadas, la aplicación del Pir aisladamente para el rastreo de ancianos con riesgo de uso elevado de los servicios de salud se ha mostrado inadecuado, especialmente debido a la baja sensibilidad.

Palabras clave: Tamizaje masivo; Exactitud de los datos; Envejecimiento; Servicios de salud.

pelo Sistema Único de Saúde (SUS) entre 2002 e 2011 e verificaram que 20 milhões e meio de hospitalizações de idosos produziram um custo de 21 bilhões e meio de reais. Os idosos representavam $16,11 \%$ dos internados, responsáveis por $28 \%$ do total das admissões e por $36,5 \%$ dos recursos pagos por elas. Observou-se também que a razão entre a porcentagem dos valores pagos para as internações e a porcentagem da população aumenta progressivamente com a idade, perfazendo uma razão de custo oito vezes mais onerosa para a população idosa em comparação à população adulta.

\section{A fragilidade e sua relação com o uso de serviços de saúde}

A fragilização não é um processo inexorável do envelhecimento; entretanto, a sua prevalência é alta, estimada entre 10 e $25 \%$ nos indivíduos com 65 anos ou mais. Ela tende a aumentar nas faixas etárias mais avançadas, de maneira que 30 a $45 \%$ dos indivíduos com 85 anos ou mais são frágeis. Em recente estudo, ${ }^{5}$ constataram-se 9,1\% de idosos frágeis na Zona Norte do Rio de Janeiro, dos quais $34,7 \%$ tinham 85 anos ou mais.

Existe uma ampla discussão sobre o conceito de fragilidade do idoso, entretanto há um consenso de que os idosos frágeis constituem um subgrupo de indivíduos com necessidade de cuidados especiais e intensivos de saúde, além de grande suporte da comunidade e da família. A provisão dos cuidados para esse crescente subgrupo é um problema crítico em saúde pública. ${ }^{6}$ A identificação precoce dos idosos frágeis ou sob risco de fragilização é uma conduta importante no manejo dessa população, pois possibilita tanto o estabelecimento de prioridades em relação à atenção a sua saúde, como o direcionamento para os centros de tratamento especializado, onde uma abordagem multidimensional e continuada visa à redução da instabilidade clínica, da incapacidade e dependência e de hospitalizações, entre outros. ${ }^{6-7}$ 


\section{Artigo de revisão}

\section{O rastreio de idosos frágeis}

Diversas combinações de diferentes critérios foram propostas por vários autores para o rastreamento de idosos frágeis. De modo geral, foram utilizadas composições distintas de indicadores de mobilidade, força muscular, dependência para as atividades de vida diária, cognição, morbidades, uso de serviços de saúde, marcadores biológicos de inflamação e estado nutricional.[8] As hospitalizações frequentes são também consideradas por alguns autores como indicadores de fragilidade.[9] Vários modelos utilizam o risco de internação hospitalar como desfecho na construção de instrumentos de rastreio de idosos portadores de risco aumentado de eventos adversos de saúde. ${ }^{8,10}$

Instrumentos breves de predição de risco para medir desfechos específicos são úteis para a identificação de indivíduos de alto risco. Uma vez identificados, os indivíduos podem ser avaliados de forma multidimensional, com o objetivo de estabelecer diagnósticos abrangentes e planejar as intervenções adequadas. Dessa forma, otimizam-se recursos e tempo. Para tal, as características psicométricas do instrumento devem ser apropriadas, com confiabilidade e acurácia adequadas, aceitáveis do ponto de vista das necessidades de gestores e equipes de profissionais da saúde. ${ }^{10}$

\section{$O$ instrumento Probabilidade de Internações Repetidas (Pir)}

Nos Estados Unidos da América (EUA), na década de 1990, Boult e colaboradores ${ }^{11}$ publicaram um instrumento cujo objetivo era o rastreamento de idosos com alto risco de internações hospitalares. Consistia em um questionário contendo oito itens, cujas respostas geravam um coeficiente de probabilidade de internações hospitalares repetidas que variava de 0 a 1 , classificando o idoso como de alto ou baixo risco, de acordo com um ponto de corte de 0,5. Trabalhos americanos, dos mesmos autores, mostravam que pessoas de alto risco internavam mais, por mais tempo e com maiores custos quando comparado com os de baixo risco.

Diante de tais resultados, Boult e colaboradores ${ }^{11}$ recomendaram o Pir como um instrumento de rastreio de indivíduos com alto risco de hospitalização, candidatos a intervenções terapêuticas e preventivas cujos objetivos seriam a redução de internações e seus custos e a melhoria da qualidade de vida. A fórmula de regressão logística, os itens utilizados para o cálculo do Pir e seus coeficientes correspondentes ${ }^{12}$ são apresentados a seguir (Figura 1 e Tabela 1).
Devido a sua fácil aplicação, tanto por telefone como por entrevista face a face, e à suposta validade preditiva e confiabilidade, esse instrumento foi indicado por representantes de operadoras de seguro de saúde americanas como um indicador primário de risco, com a proposta de uma busca proativa desses idosos, para que fossem encaminhados a programas especializados. ${ }^{13}$ Como o instrumento também tem sido proposto para uso no Brasil, pela Agencia Nacional de Saúde Suplementar (ANS), faz-se necessário conhecer o

$$
\begin{aligned}
& \text { Pir }=\quad \frac{\mathrm{e}^{\mathrm{Bx}}}{1+\mathrm{e}^{\mathrm{B} \mathrm{x}}} \\
& \text { Pir }=\text { Probabilidade de internações repetidas em } 4 \text { anos } \\
& \text { e }=\text { logaritmo natural }=2,7183 \\
& \beta x=\beta_{0}+\sum^{13} y=1 \beta y X y, \text { onde: } \\
& \beta_{0}=(-1.802) \text {, uma constante da equação da regressão logística } \\
& \beta y=\text { coeficiente de regressão logística de cada fator de risco } \\
& \mathrm{X} y=1 \text { ou } 0 \text { segundo a presença ou ausência de cada fator de risco }
\end{aligned}
$$

Figura 1. Número de estudos identificados selecionados para inclusão na revisão sistemática e os motivos da exclusão.

Tabela 1. Variáveis utilizadas para o cálculo da Probabilidade de internações repetidas e os coeficientes de regressão correspondentes.

\begin{tabular}{|l|c|}
\hline \multicolumn{1}{|c|}{ Variáveis (y) } & $\begin{array}{c}\text { Coeficientes de } \\
\text { regressão (By) }\end{array}$ \\
\hline 1. Autoavaliação da saúde: muito boa & 0,327 \\
\hline 2. Autoavaliação da saúde: boa & 0,340 \\
\hline 3. Autoavaliação da saúde: regular & 0,552 \\
\hline 4. Autoavaliação da saúde: ruim & 0,770 \\
\hline 5. Ter doença coronariana & 0,390 \\
\hline 6. Ter diabetes mellitus & 0,319 \\
\hline 7. Ter estado internado no último ano & 0,545 \\
\hline 8. Número de consultas médicas no & 0,318 \\
\hline último ano > 6 & $-0,738$ \\
\hline 9. Não ter cuidador informal & 0,255 \\
\hline 10. Idade: $75-79$ & 0,327 \\
\hline 11. Idade: $80-84$ & 0,559 \\
\hline 12. Idade: 85 ou + & 0,257 \\
\hline 13. Sexo masculino & \\
\hline
\end{tabular}

Fonte: Veras, 2002 
seu desempenho em predizer desfechos desfavoráveis de saúde em nosso meio. ${ }^{14}$

O objetivo desse trabalho é provocar uma reflexão sobre a utilidade do Pir a partir dos dados sobre a sua acurácia.

\section{Métodos}

Revisão narrativa de estudos originais de validação preditiva, de revisões sistemáticas internacionais com e sem metanálise e de todos os estudos brasileiros que utilizaram o Pir. Realizada busca nas bases de dados PubMed, Bireme e Google Acadêmico utilizando as palavras-chave "Envelhecimento", "Idoso fragilizado", "Rastreamento", "Hospitalização", "Sensibilidade e Especificidade".

\section{Desenvolvimento}

Durante quase duas décadas, Boult e colaboradores[15-16] publicaram um número variado de trabalhos que reafirmaram a validade do Pir para estratificação de risco de diferentes populações, em distintos cenários, utilizando-o, ainda, em vários ensaios clínicos para testar modelos de gerenciamento de cuidados. O Pir também foi submetido a processos de validação em alguns países europeus, como Inglaterra, Alemanha e Suíça. ${ }^{17}$

Em 1993, no estudo original, ${ }^{11}$ a acurácia calculada através da área sob a curva ROC (Receiver Operator Characteristic) foi de $61 \%$ para internações repetidas. Embora a sensibilidade, especificidade e os valores preditivos positivo e negativo não estivessem disponíveis no estudo original, calculou-se o seu valor a partir dos dados fornecidos no artigo de Boult e colaboradores, obtendo-se sensibilidade de $12,7 \%$ e especificidade de $94,6 \%$; e valor preditivo positivo de $42 \%$ e negativo de $78 \%$. Mesmo com esse desempenho, os autores consideraram o instrumento como tendo boa acurácia e, portanto, válido para o rastreio de indivíduos frágeis.

Em uma recente revisão sistemática, O'Caoimh e colaboradores ${ }^{10}$ selecionaram 23 instrumentos, com o objetivo de avaliar quais eram válidos para o rastreio de idosos com alto risco de quatro desfechos desfavoráveis: hospitalização, declínio funcional, institucionalização e morte. Entre todos, o instrumento Pir foi o mais estudado quanto à validade externa. Contudo, os autores concluíram que nenhum deles mostrou acurácia adequada para ser usado isoladamente.

Em 2013, Wallace e colaboradores ${ }^{18}$ estimaram uma acurácia de 69,7\%, no resultado de uma metanálise de cinco coortes, totalizando quase nove mil indivíduos, revelando uma modesta capacidade de discriminação do modelo, além de uma alta especificidade sumária (95\%, IC: 95,8 a 96,7\%). Apesar desses dados, os autores sugeriram que o instrumento seria de utilidade clínica, e também para gestão e planejamento de saúde; no entanto, alertaram para o problema da quantidade elevada de falsos negativos produzidos pela baixa sensibilidade (12\%). O mesmo grupo de autores publicou um estudo, em 2016, ${ }^{19}$ com o objetivo de reavaliar a validade externa do instrumento, analisando 862 idosos de uma amostra comunitária na Irlanda; encontraram 7\% de indivíduos de alto risco e a área sob a curva (AUC) de 0,65 para internações de emergência. Concluíram que o instrumento teve um baixo poder de discriminação, identificou uma pequena proporção de idosos de alto risco, mas apresentou alta especificidade. Sugeriram modificações no instrumento, com a incorporação de variáveis de medidas de morbidade, na tentativa de melhorar a sua acurácia.

\section{A experiência brasileira no rastreio de idosos com alto risco de internações}

No Brasil, o Pir foi apresentado com o nome de Triagem Rápida, ${ }^{12}$ tendo sido testado inicialmente em 2002, em 360 indivíduos de um ambulatório da rede pública, para captar idosos com alto risco de desfechos adversos de saúde. O objetivo era discutir uma proposta de fluxo de acesso e definição de prioridades em uma rede ambulatorial. Nesse trabalho, surgiu a proposta de pontos de corte intermediários para uma maior estratificação de risco (baixo, médio, médio-alto e alto). Evidenciou-se que 75\% dos idosos apresentavam baixo risco de internação e $11 \%$ apresentavam risco médio a alto, com maior potencial de uso de serviço de saúde, e que, portanto, deveriam ter o atendimento priorizado. Concluiu-se que os resultados de análise transversal sugeriam ser o Pir eficaz para classificar e estratificar os idosos em diferentes grupos de risco, possibilitando a hierarquização da atenção à saúde dessa população. Entretanto, ao final, apontou-se para a necessidade de estudos longitudinais em nosso meio para a validação preditiva do instrumento.

OPir também foi estudado na cidade de João Neiva (ES), em 2004, em duas unidades de saúde, uma urbana e outra rural.20 Foram entrevistados e estratificados 103 idosos e os resultados mostraram um maior risco de internação em indivíduos da área rural $(54,5 \%)$, quando comparados com os da área urbana (42,4\%). Mais uma vez o instrumento foi considerado útil para identificar idosos sob risco e os autores ainda propuseram a sua 


\section{Artigo de revisão}

Tabela 2. Publicações brasileiras que utilizaram o instrumento Probabilidade de Internações Repetidas

\begin{tabular}{|c|c|c|c|c|c|c|c|}
\hline $\begin{array}{l}\text { Referência } \\
\text { bibliográfia }\end{array}$ & Cidade & Cenário & Ponto de corte & $\begin{array}{c}\text { Tamanho da } \\
\text { amostra }\end{array}$ & $\begin{array}{c}\text { Desenho do } \\
\text { estudo }\end{array}$ & Objetivo & Publicação \\
\hline $\begin{array}{l}\text { Veras et al. } \\
2002^{12}\end{array}$ & $\begin{array}{l}\text { Rio de Janeiro } \\
\text { (RJ) }\end{array}$ & $\begin{array}{l}\text { Policlínica da } \\
\text { Universidade } \\
\text { do Estado do } \\
\text { Rio de Janeiro } \\
\text { (guichê de } \\
\text { atendimento) }\end{array}$ & $\begin{array}{c}<0,30 \\
0,30-0,39 \\
0,40-0,49 \\
\geq 0,50\end{array}$ & 360 & Transversal & $\begin{array}{c}\text { Estratificação de } \\
\text { risco }\end{array}$ & $\begin{array}{l}\text { Livro Terceira } \\
\text { idade: gestão } \\
\text { contemporânea } \\
\text { em saúde }\end{array}$ \\
\hline Veras, $2003^{24}$ & $\begin{array}{c}\text { Rio de Janeiro } \\
\text { (RJ) }\end{array}$ & $\begin{array}{l}\text { Policlínica da } \\
\text { Universidade } \\
\text { do Estado do } \\
\text { Rio de Janeiro } \\
\text { (guichê de } \\
\text { atendimento) }\end{array}$ & $\begin{array}{c}<0,30 \\
0,30-0,39 \\
0,40-0,49 \\
\geq 0,50\end{array}$ & 360 & Revisão & $\begin{array}{l}\text { Proposta } \\
\text { de modelo } \\
\text { assistencial }\end{array}$ & $\begin{array}{l}\text { Cadernos de } \\
\text { Saúde Pública }\end{array}$ \\
\hline $\begin{array}{l}\text { Negri et al. } \\
2004^{20}\end{array}$ & João Neiva (ES) & $\begin{array}{l}\text { Unidades } \\
\text { Básicas de } \\
\text { Saúde - duas } \\
\text { (ambulatóri) }\end{array}$ & $\begin{array}{c}<0,30 \\
0,30-0,39 \\
0,40-0,49 \\
\geq 0,50\end{array}$ & 103 & Transversal & $\begin{array}{c}\text { Estratificação de } \\
\text { risco }\end{array}$ & $\begin{array}{l}\text { Ciência \& } \\
\text { Saúde Coletiva }\end{array}$ \\
\hline $\begin{array}{l}\text { Lourenço et al. } \\
2005^{25}\end{array}$ & $\begin{array}{c}\text { Rio de Janeiro } \\
(\mathrm{RJ})\end{array}$ & NA & $\begin{array}{c}<0,30 \\
0,30-0,39 \\
0,40-0,49 \\
\geq 0,50\end{array}$ & NA & $\begin{array}{l}\text { Descrição de } \\
\text { um modelo } \\
\text { assistencial }\end{array}$ & $\begin{array}{l}\text { Proposta } \\
\text { de modelo } \\
\text { assistencial }\end{array}$ & $\begin{array}{l}\text { Revista de } \\
\text { Saúde Pública }\end{array}$ \\
\hline $\begin{array}{l}\text { Guerra et al. } \\
2007^{21}\end{array}$ & Botucatu (SP) & $\begin{array}{l}\text { Centro de } \\
\text { Saúde Escola de } \\
\text { Botucatu } \\
\text { (ambulatório) }\end{array}$ & $\begin{array}{c}<0,30 \\
0,30-0,39 \\
0,40-0,49 \\
\geq 0,50\end{array}$ & 305 & Transversal & $\begin{array}{l}\text { Estratificação de } \\
\text { risco }\end{array}$ & $\begin{array}{l}\text { Caderno de } \\
\text { Saúde Pública }\end{array}$ \\
\hline $\begin{array}{l}\text { Estrella et al. } \\
2009^{22}\end{array}$ & $\begin{array}{c}\text { Rio de Janeiro } \\
\text { (RJ) }\end{array}$ & $\begin{array}{l}\text { Clientes de } \\
\text { operadora } \\
\text { de saúde } \\
\text { (telefone) }\end{array}$ & $\begin{array}{c}<0,30 \\
0,30-0,39 \\
0,40-0,49 \\
\geq 0,50\end{array}$ & 2.637 & Transversal & $\begin{array}{l}\text { Estratificação de } \\
\text { risco e proposta } \\
\text { de modelo }\end{array}$ & $\begin{array}{l}\text { Caderno de } \\
\text { Saúde Pública }\end{array}$ \\
\hline $\begin{array}{l}\text { Dutra et al. } \\
2011^{23}\end{array}$ & Progresso (RS) & $\begin{array}{l}\text { Estratégia } \\
\text { da Saúde da } \\
\text { Família } \\
\text { (domicílio) }\end{array}$ & $\begin{array}{c}<0,30 \\
0,30-0,39 \\
0,40-0,49 \\
\geq 0,50\end{array}$ & 515 & $\begin{array}{c}\text { Coorte } \\
\text { prospectivo }\end{array}$ & $\begin{array}{l}\text { Validação } \\
\text { preditiva }\end{array}$ & $\begin{array}{l}\text { Revista de } \\
\text { Saúde Pública }\end{array}$ \\
\hline $\begin{array}{l}\text { Berlezi et al. } \\
2011^{26}\end{array}$ & Ijui (RS) & $\begin{array}{l}\text { Estratégia } \\
\text { da Saúde da } \\
\text { Família } \\
\text { (domicílio) }\end{array}$ & $\begin{array}{c}<0,30 \\
0,30-0,39 \\
0,40-0,49 \\
\geq 0,50\end{array}$ & NA & Relato de caso & $\begin{array}{l}\text { Proposta de mo- } \\
\text { delo assistencial }\end{array}$ & $\begin{array}{l}\text { Texto \& } \\
\text { Contexto de } \\
\text { Enfermagem }\end{array}$ \\
\hline $\begin{array}{l}\text { Perez et al. } \\
2013^{27}\end{array}$ & $\begin{array}{c}\text { Rio de Janeiro } \\
(\mathrm{RJ})\end{array}$ & $\begin{array}{l}\text { Clientes de } \\
\text { operadora } \\
\text { de saúde } \\
\text { (domicílio) }\end{array}$ & $\geq 0,50$ & 764 & Transversal & $\begin{array}{l}\text { Estratificação de } \\
\text { risco \& fatores } \\
\text { associados }\end{array}$ & $\begin{array}{l}\text { Caderno de } \\
\text { Saúde Pública }\end{array}$ \\
\hline Oliveira, $2015^{28}$ & João Pessoa (PB) & $\begin{array}{l}\text { Estratégia } \\
\text { da Saúde da } \\
\text { Família } \\
\text { (domicílio) }\end{array}$ & $\begin{array}{c}<0,30 \\
0,30-0,39 \\
0,40-0,49 \\
\geq 0,50\end{array}$ & 368 & Transversal & $\begin{array}{l}\text { Estratificação de } \\
\text { risco \& fatores } \\
\text { associados }\end{array}$ & $\begin{array}{l}\text { Dissertação de } \\
\text { mestrado } \\
\text { Centro de Ciên- } \\
\text { cias da Saúde } \\
\text { da Universida- } \\
\text { de Federal da } \\
\text { Paraíba }\end{array}$ \\
\hline
\end{tabular}

Legenda: NA: Não se aplica. 
aplicação no planejamento de serviços primários de saúde, sugerindo, ainda, a partir dos dados encontrados, a criação de um programa específico para atendimento aos idosos fragilizados naquela cidade.

Em 2007, em outro trabalho de delineamento transversal, Guerra e colaboradores ${ }^{21}$ estratificaram, segundo o risco de internação, uma amostra de idosos atendidos no Centro de Saúde Escola da Faculdade de Medicina de Botucatu. Os 305 pacientes avaliados com o Pir foram classificados com seguinte perfil de risco: baixo: $56,4 \%$; médio: 26,9\%; médio-alto: 10,5\%; e alto: 16,2\%.

Em 2005, Estrella e colaboradores22 testaram a proposta de hierarquização da demanda de idosos do sistema de saúde complementar a partir do perfil do risco de internação hospitalar. O instrumento foi aplicado por telefone, pelo setor de call center, a 2637 clientes de uma operadora de saúde da cidade do Rio de Janeiro, e estimou-se que 76,0\% dos idosos eram de baixo risco, $13,4 \%$ eram de médio risco, 7,23\% eram de risco médio-alto e 3,23\% eram de alto risco de internações repetidas. Os autores sugeriram o uso de tais categorias para o planejamento de programas de saúde de diferentes níveis de intervenção, por meio de implementação de medidas preventivas ou assistenciais específicas.

O único estudo brasileiro longitudinal de validação preditiva do Pir disponível na literatura foi publicado em 2011 por Dutra e colaboradores. ${ }^{23}$ Os 515 indivíduos com 60 anos ou mais, residentes na comunidade de Progresso (RS), foram estratificados e acompanhados por seis meses. Utilizou-se a análise de sobrevida para avaliar a probabilidade de internação hospitalar entre os idosos dos diferentes estratos de risco. A acurácia foi estudada mediante a determinação da área sob a curva ROC. Os idosos de alto risco foram internados 6,5 mais vezes do que os de baixo risco, e a acurácia foi de 67\%. Apesar do curto tempo de seguimento, o estudo mostrou alguma evidência da capacidade preditiva do instrumento em nosso meio.

A Tabela 2 resume as publicações brasileiras que utilizaram o Pir. Como descrito acima, trata-se, principalmente, de trabalhos com delineamento transversal, descritivos; outros são propostas de modelo assistencial para os pacientes idosos de vários municípios em diferentes estados da federação, nos quais o instrumento é utilizado para estratificação de risco e organização da demanda. Há, portanto, uma escassez de estudos longitudinais para a validação preditiva no Brasil.

\section{Discussão}

A presente revisão constatou que em todos os estu- dos que avaliaram as características psicométricas do Pir, incluindo a sua primeira publicação, a sensibilidade mostrou-se baixa, a despeito de alta especificidade e que, até o momento, não existem muitas evidências de seu desempenho na população brasileira.

A escolha de um instrumento a ser utilizado em programas de gestão de saúde tem implicações financeiras e éticas. O rastreamento se justifica caso identifique os indivíduos em uma fase inicial do processo de adoecimento, quando intervenções preventivas são possíveis. Para isso, a sensibilidade do teste é uma propriedade importante. ${ }^{29}$ Segundo Fletcher \& Fletcher ${ }^{29}$ "a sensibilidade e a especificidade são as propriedades que deveriam ser levadas em consideração quando se decide usar ou não um teste". Esses autores ressaltam essas características porque a inadequação psicométrica pode ter como consequência o desperdício de recursos com indivíduos falso-positivos, mas também a exclusão de indivíduos falso-negativos, candidatos às intervenções específicas. Além disso, ao se utilizar um instrumento com alta especificidade, arrisca-se captar idosos em um ponto já avançado da trajetória da fragilidade, no qual intervenções preventivas não sejam mais efetivas.

Apesar de algumas evidências de sua utilidade para o Brasil, o Pir foi criado a partir da identificação de variáveis preditivas baseadas em dados empíricos de amostras da população norte-americana. Avaliar a sua composição de itens e se o escore de risco do modelo tem valor preditivo semelhante para uma realidade socioeconômica e cultural distinta da americana é uma etapa importante, anterior à sua utilização sistemática, uma vez que a assunção a priori da universalidade do instrumento pode induzir a erros graves de estratificação de risco dos indivíduos.

A busca por um instrumento adequado (com boa acurácia) para o rastreio de idosos continua atual, a despeito das várias ferramentas propostas nas últimas três décadas. Essa necessidade se justifica pelo desafio que gestores enfrentam, em diferentes países e regiões com situação econômica diversa, para encontrar soluções que viabilizem o cuidado apropriado da saúde dessa população.

\section{Conclusão}

Embora utilizado há algumas décadas, a aplicação do Pir isoladamente para rastreio de idosos frágeis e com risco de uso elevado dos serviços de saúde tem se mostrado inadequado, especialmente devido a sua baixa sensibilidade.

No Brasil, há poucas evidencias da utilidade do 


\section{Artigo de revisão}

instrumento, mas em breve serão disponibilizados os resultados de um estudo longitudinal de validação preditiva utilizando o Pir.

\section{Referências}

1. Banco Mundial. Envelhecendo em um Brasil mais Velho: implicações do envelhecimento populacional para o crescimento econômico, a redução da pobreza, as finanças públicas e a prestação de serviços. Sumário Executivo. [S.I.]: Banco Mundial, 2011. Disponível em: <http://siteresources.worldbank.org/BRAZILINPOREXTN/ Resources//3817166-1302102548192/Envelhecendo_Brasil_ Sumario_Executivo.pdf>. Acesso em: 13/03/2017.

2. World Health Organization. World Report on Ageing and Health. [S.I.]: World Health Organization, 2015. Acesso em: 02/05/2016. Disponível em: <http://www.who.int/ageing/ publications/world-report-2015/en/>.

3. La Forgia GM, Couttolenc BF. Hospital Performance in Brazil: The Search for Excellence. Washington, DC: World Bank, 2008. Acesso em: 10/11/2016. Disponível em: <https:// openknowledge.worldbank.org/handle/10986/6516>.

4. Silveira RE, Santos AS, Sousa MC, et al. Gastos relacionados a hospitalizações de idosos no Brasil: perspectivas de uma década. Einstein. 2013;11(4):514-520.

5. Moreira VG, Lourenço RA. Prevalence and factors associated with frailty in an older population from the city of Rio de Janeiro, Brazil: the FIBRA-RJ Study. Clinics. 2013;687(7):979-985.

6. Fried LP, Tangen CM, Walston J, et al. Frailty in older adults: evidence for a phenotype. J Gerontol A Biol Sci Med Sci. 2001;56(3):M146-56.

7. Veras R. Em busca de uma assistência adequada à saúde do idoso: revisão da literatura e aplicação de um instrumento de detecção precoce e de previsibilidade de agravos. Cad. Saúde Pública. 2003;19:705-715.

8. Hogan DB, Macknight C, Bergman H. Models, definitions, and criteria of frailty. Aging Clin Exp Res. Jun 2003;15(Supl 3):1-29.

9. Pacala JT, Boult C, Boult L. Predictive validity of a questionnaire that identifies older persons at risk for hospital admission. J Am Geriatr Soc. Apr 1995;43(4):374-377.

10. O'Caoimh R, Cornally N, Weathers E, et al. Risk prediction in the community: A systematic review of case-finding instruments that predict adverse healthcare outcomes in community-dwelling older adults. Maturitas. Sep 2015; 82(1): 3-21.

11. Boult C, Dowd B, Mccaffrey D, et al. Screening elders for risk of hospital admission. J Am Geriatr Soc. Aug 1993;41(8):811-817.

12. Veras RP, Lourenço RA, Martins CSF, et al. Novos paradigmas do modelo assistencial no setor saúde: consequência da explosão populacional dos idosos no Brasil. In: Veras, RP (Org). Terceira idade: gestão contemporânea em saúde, Rio de Janeiro: Relume-Dumará; 2002. p.11-81.

13. Sidorov J, Shull R. "My patients are sicker:" using the Pra risk survey for case finding and examining primary care site utilization patterns in a medicare-risk MCO. Am J Manag Care. Jun 2002;8(6):569-575.

14. Agência Nacional de Saúde Suplementar. Idoso na saúde suplementar: uma urgência para a saúde da sociedade e para a sustentabilidade do setor: Projeto Idoso Bem Cuidado. Rio de Janeiro: Agência Nacional de Saúde Suplementar, 2016. 131 p. Acesso em: 23/04/2016. Disponível em: <http://www. ans.gov.br/images/stories/Materiais_para_pesquisa/Materiais_ por_assunto/web_final_livro_idosos.pdf>.

15. Boult C, Boult L, Murphy C, et al. A controlled trial of outpatient geriatric evaluation and management. J Am Geriatr Soc. May 1994;42(5):465-470.

16. Boult C, Boult LB, Morishita L, et al. A randomized clinical trial of outpatient geriatric evaluation and management. J Am Geriatr Soc. Apr 2001;49(4):351-359.

17. Wagner JT, Machmann LM, Boult C, et al. Predicting the risk of hospital admission in older persons--validation of a brief selfadministered questionnaire in three European countries. J Am Geriatr Soc. 2006;54:1271-1276.

18. Wallace E, Hinchey T, Dimitrov BD, et al. A systematic review of the probability of repeated admission score in communitydwelling adults. J Am Geriatr Soc. Mar 2013;61(3):357-364.

19. Wallace E, Mcdowell R, Bennett K, et al. External validation of the Probability of repeated admission (Pra) risk prediction tool in older community-dwelling people attending general practice: a prospective cohort study. BMJ Open. Nov 2016;6(11):e012336.

20. Negri LDSA, Ruy GF, Collodetti JB, et al. Aplicação de um instrumento para detecção precoce e previsibilidade de agravos na população idosa. Ciênc. saúde coletiva. 2004;9(4):1033-1046.

21. Guerra IC, Ramos-Cerqueira ATDA. Risco de hospitalizações repetidas em idosos usuários de um centro de saúde escola. Cad. Saúde Pública. Mar 2007;23:585-592.

22. Estrella K, Oliveira CE, Sant'anna AA, et al. Screening for risk of hospitalization in the elderly: a study based on a single entry point in a health maintenance organization in Brazil. Cad. Saúde Pública. Mar 2009;25(3):507-512.

23. Dutra MM, Moriguchi EH, Lampert MA, et al. Validade preditiva de instrumento para identificação do idoso em risco de hospitalização. Rev. Saúde Pública. 2011;45(1):106-112.

24. Veras R. Em busca de uma assistência adequada à saúde do idoso: revisão da literatura e aplicação de um instrumento de detecção precoce e de previsibilidade de agravos. Cad. Saúde Pública. 2003;19:705-715.

25. Lourenço RA, Martins CSF, Sanchez MAS, et al. Assistência ambulatorial geriátrica: hierarquização da demanda. Rev. Saúde Pública. 2005;39(2):311-318.

26. Berlezi EM, Eickhoff HM, Oliveira KR, et al. Programa de atenção ao idoso: relato de um modelo assistencial. Texto contexto - enferm. 2011;20(2):368-370.

27. Perez M, Lourenço RA. Rede FIBRA-RJ: fragilidade e risco de hospitalização em idosos da cidade do Rio de Janeiro, Brasil. Cad. Saúde Pública. 2013;29(7):1381-1391.

28. Oliveira FMRL. Risco de hospitalização repetida em idosos atendidos na atenção primária de saúde de João Pessoa, Paraíba [Dissertação]. João Pessoa: Universidade Federal da Paraíba; 2015.

29. Fletcher RW, Flectcher SE. Epidemiologia clínica: elementos essenciais. 4. ed. [S.I.]: Artmed, 2006. 